Rev. Elev. Méd. vét. Pays trop. 1967, 20, 2 (261-271)

\title{
Les vecteurs de Stilesia globipunctata (Rivolta, 1874) du mouton
}

\author{
par M. GRABER et J. GRUVEL \\ (Avec la collaboration technique de Madame BRUNET)
}

\begin{abstract}
RÉSUME
Les auleurs ont réussi à transmettre Stilesıo globipunctoto (RIVOLTA, 1874) à des moutons préalablement déparasttés (63) ò partir des Oribatcs suivants : Scheloribotes perforatus (WALLWORK, 1964), Scheloribates parvus Van Pletzen conglobotus (WALLWORK, 1964), Scheloribotes fimbriotus Thor africonus (WALLWORK, 1964), Golumno bologhi (WALLWORK, 1965), Allogalumno pellucido (WALLWORK, 1965) et Africocarus coicorotus (WALLWORK, 1965).

II existe dans les pâturages, chaque mois, au minimum et au maximum cinq espèces susceptibles d'héberger les Cysticercordes de cet Ancplocephalidoe, ce qui assure la pérennité et la constance de l'infestation chez les ovins.

Les malleurs vecteurs semblent appartenır à la famil'e des Galumnidae. Scheloribates perforatus compense un taux d'infestahion relativement faible par une large dispersion ef une grande abondance, ce qui donne au mouton, hôte définitif, la possibilité de l'absorber plus fréquemment que les auires espèces pouvant servir de vecteurs
\end{abstract}

\section{INTRODUCTION}

Parmi les Cestodes ovins de la région de FortLamy, Strlesia globipunctata (RIVOLTA, 1874) est l'espèce dominante, puisqu'elle touche de $56 \mathrm{p}$. 100 des 2.400 moutons autopsiés jusqu'à ce jour. Des essais d'infestations expérimentales ont démontré que ce Cestode étalt transmis par des Acariens Oribates. Ce sont :

- pour les Indes (TANDON, 1963) les genres Erythacus ef Scheloribates (Scheloribates indica).

- pour le Tchad (GRABER et GRUVEL, 1964) Scheloribates perforatus (WALLWORK, 1964). Scheloribates parvus Van PLETZEN conglobatus (WALLWORK, 1964) et Africacarus calcaratus (WALLWORK, 1965).

Depuis deux ans, de nombreuses récoltes effectuées dans la concession du Laboratoire de Farcha ou dans ses environs immédiats ont permis de metire en évidence de nouvelles espèces d'Oribates susceptıbles de servir de vecteur à cet Anoplocephalidoe.

\section{MATÉRIEL ET MÉTHODE}

\section{Lieux de récolte.}

Les Acariens qui ont été soumis - sans succès pour l'instant - à des infestations par Moniezia expanso et Avitellina centripunctata ont été recueillis :

- à l'extérieur de la concession du Laboratoire de Farcha $\left(Z_{1}\right)$, dans une zone basse fréquentée par des moutons dont beaucoup étalent atteints de Téniasis. Les Acariens rencontrés appartenaient aux espèces Unguizetes reticulotus (WALLWORK, 1965), Golumna baloghı (WALLWORK, 1965), Allogalumna pellucida (WALLWORK, 1965 et Africacarus calcaratus (WALLWORK, 1965).

- dans le périmètre de la concession de Farcha.

soit à partir d'un terrain fraıs et humide, couvert de pourplers et où la densité d'Oribates était considérable $\left(Z_{2}\right)$; 


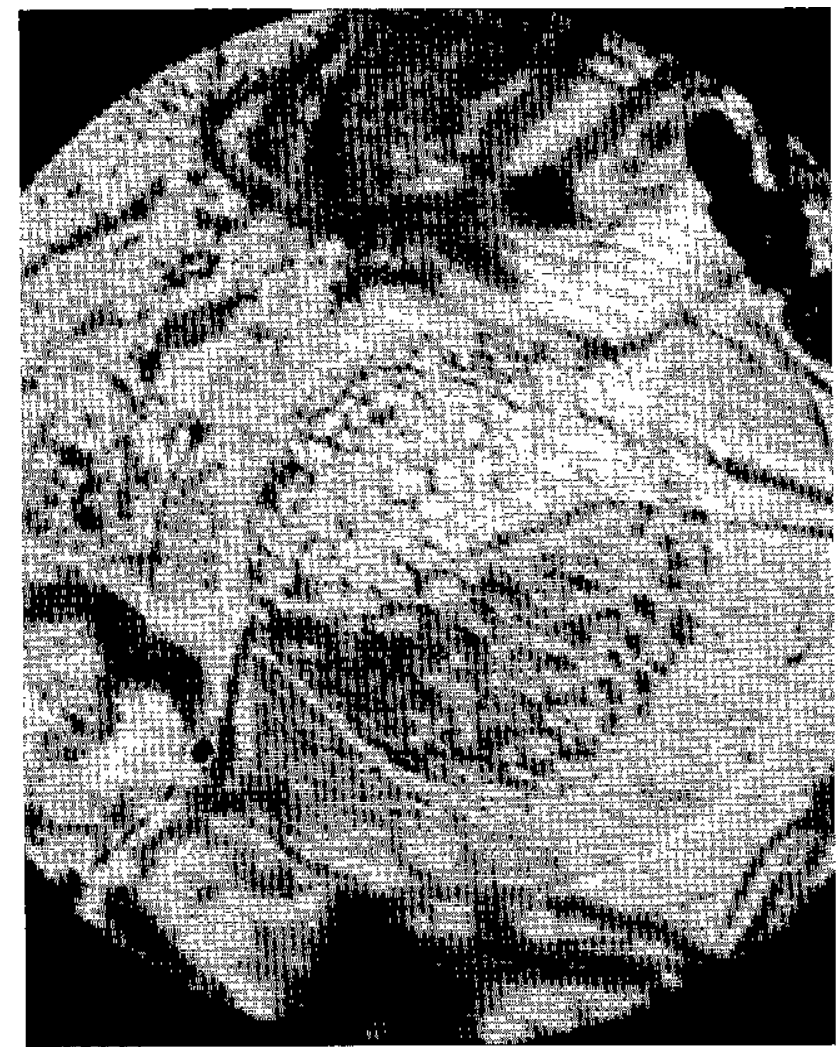

Fig. 1. - Scheloribates perforotus cysticercorde S. giobıunctato grossissement 397.

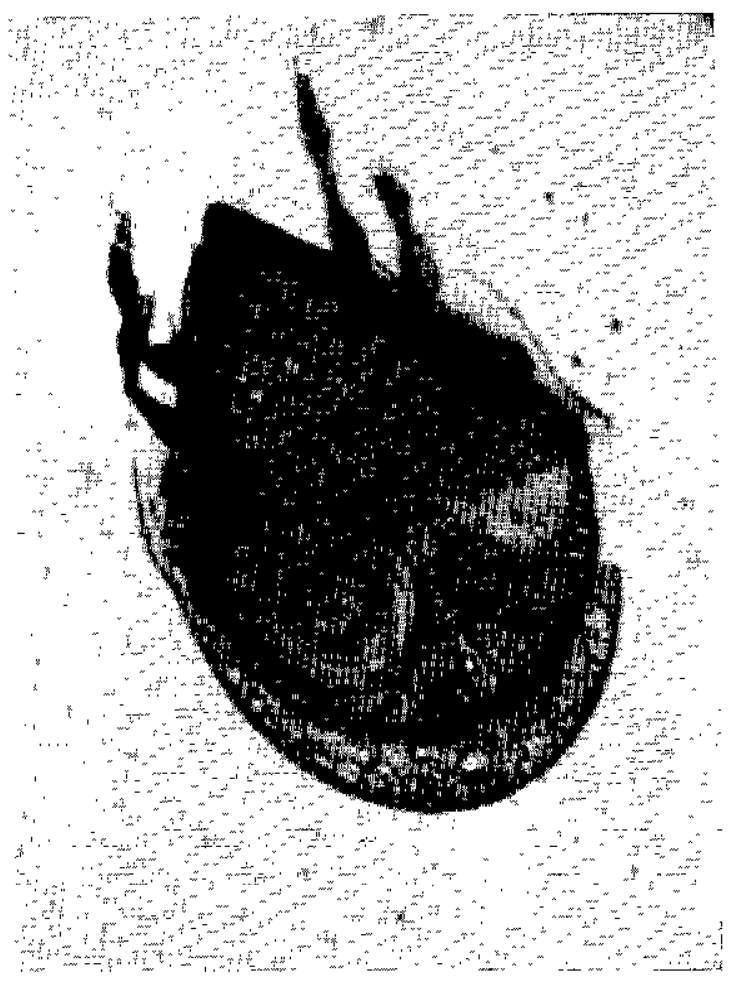

Fig. 2. - Scheloribates perforotus cysticercoide S. globipunctala grossissement 113. 


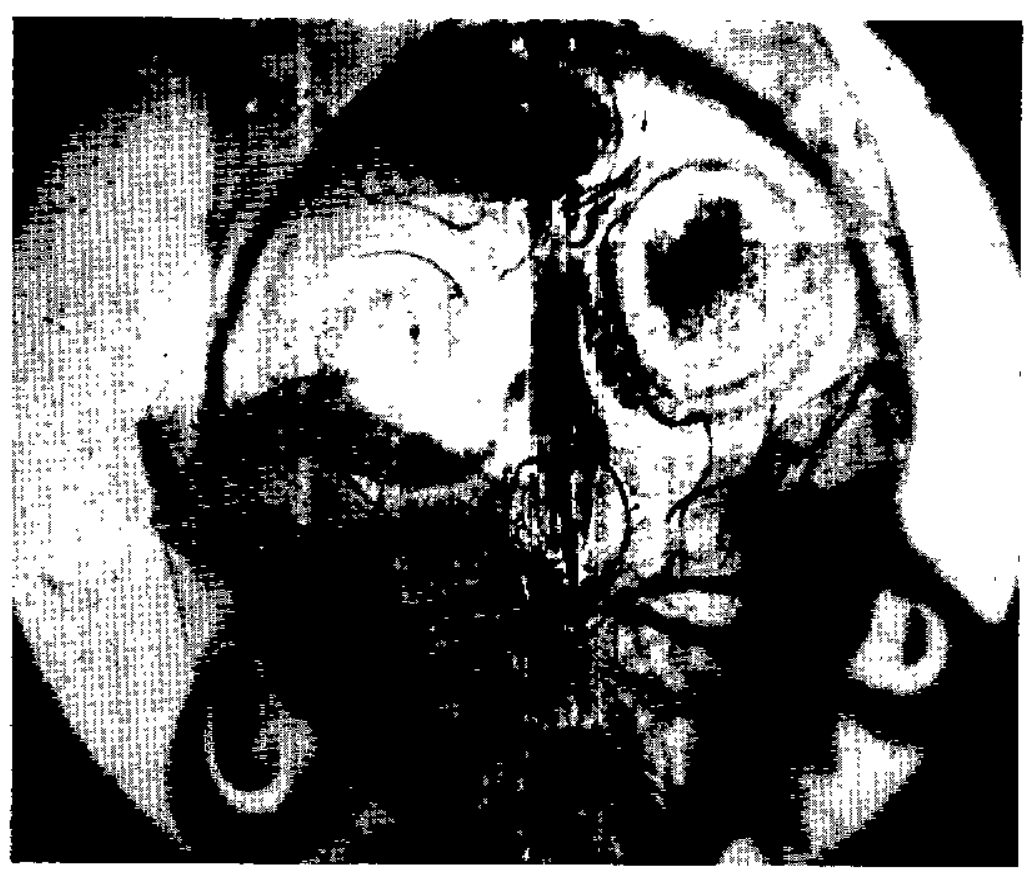

Fig. 3. - Scheloribates perforatus 2 cysticercoides 5. glopipunctato grossissement 180 .

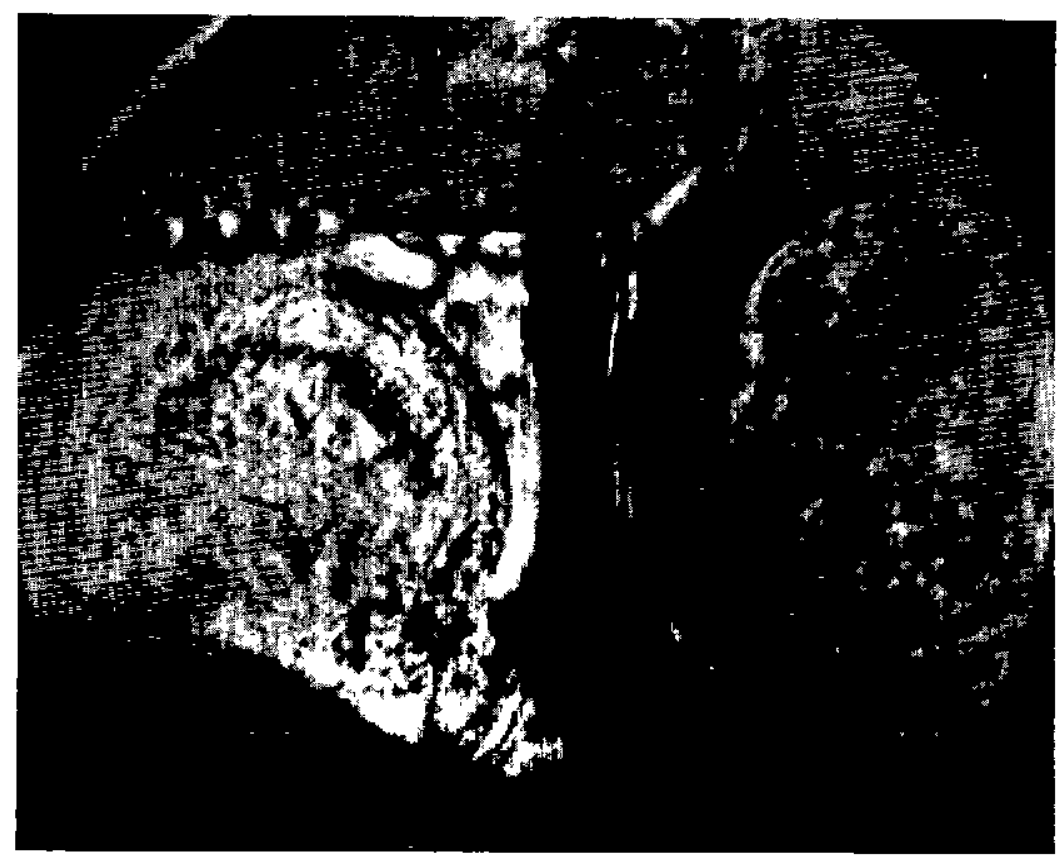

Fig. 4. - Scheloribotes perforatus 2 cysticercoides

S. globipunctota grossissement 358 . 


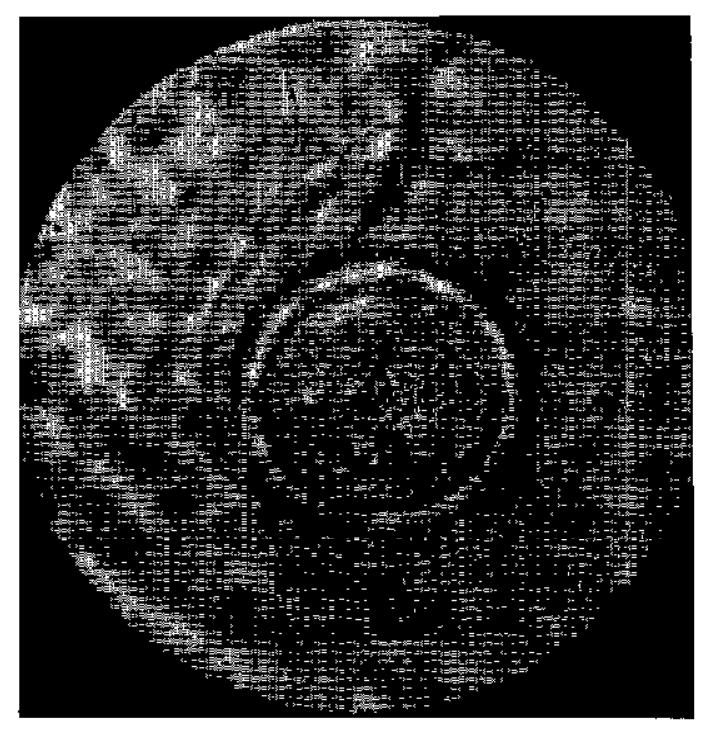

Fig. 5. - Cystıcercoide S. globipunctata

extrait d'un Columnidae. On aperçait les 4 ventouses.

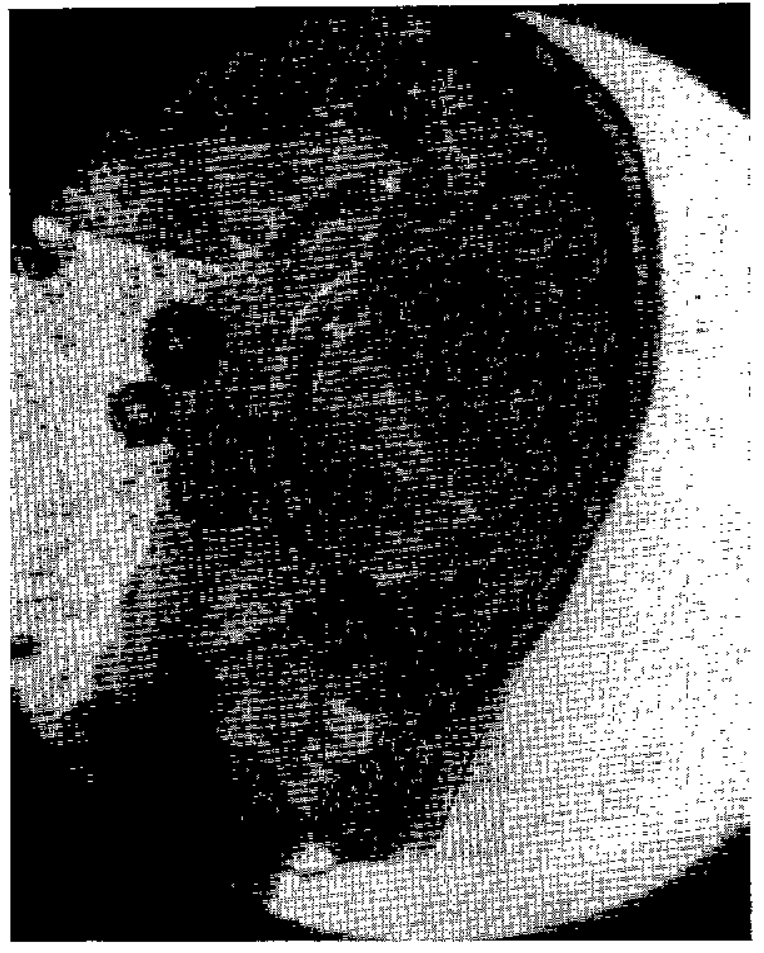

Fig. 6. - Scheloribates fimbriatus

avec un cysticercoïde $S$, globipunctata.

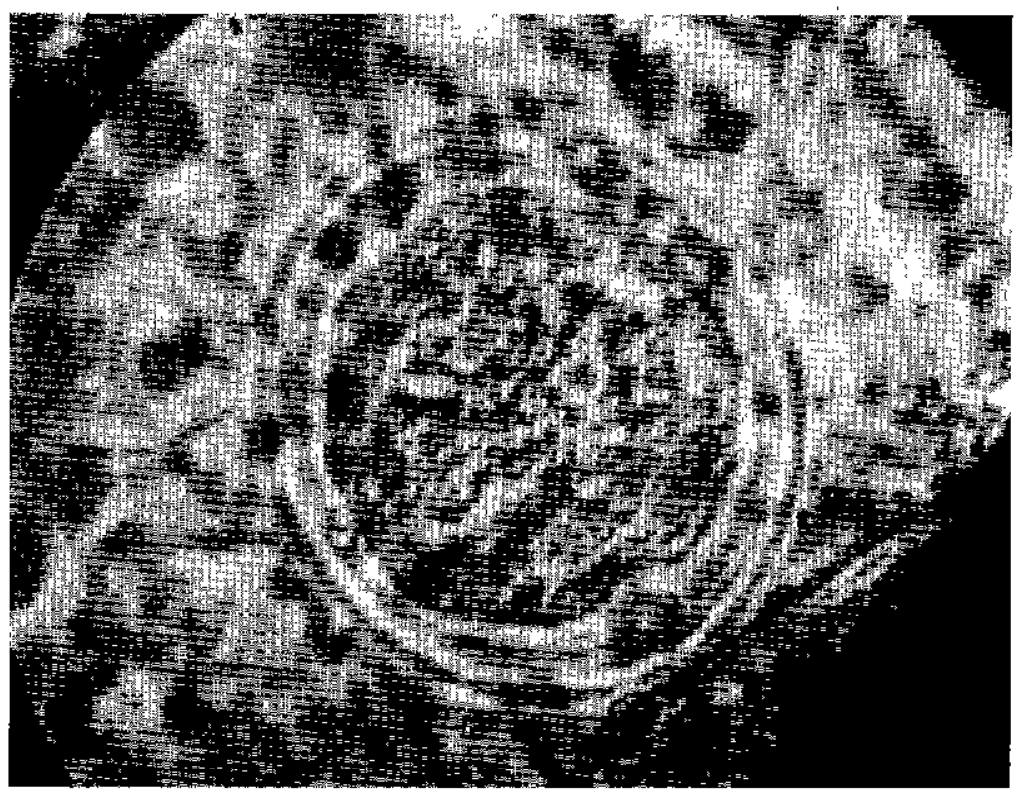

Fig. 7. - Scheloribates fimbriatus avec un cysticercoide S. globipunctato vu à un plus fort grossissement. 
soit à partır des bacs d'élevage $\left(Z_{3}\right)$ repeuplés artificiellement au moyen d'Oribates prélevés en $Z_{2}$. Ont été ainsi reconnus : Scheloribotes perforotus (WALLWORK, 1964), Scheloribates fimbriatus Thor africanus (WALLWORK, 1964), Africocorus calcarotus (WALLWORK, 1965), Golumna baloghi (WALLWORK, 1965) et Allogolumno pellucida (WALLWORK, 1965).

Beaucoup de ces Oribates, après dissection, présentaient de 1 à 4 Cysticercoides bien caractérisés, ainsi que l'indique la série de photographies jointes au présent document.

\section{Les animaux d'expérience.}

Les moutons ayant servi à l'infestation expérimentale ont été préalablement débarrassés de tous leurs Cestodes (GRABER et GRUVEL, 1964) par l'admınistration différée d'Arséniate d'étain et de 14. 015 R. P. ou de Yomesan Bayer sous forme de Mansonil. Ces taenicides sont très actifs et permettent l'expulsion de tous les Anoplocephalidae associés, y compris Stilesia globipunctata .
Les ovıns ainsi préparés ont été placés dans des stalles cimentées et nourris, dès leur arrivée, avec du fourrage vert coupé sur les bords du fleuve Chari, dans une régıon où il n'existe ni ruminants domestiques, ni ruminants sauvages. Des branches d'épineux dont les moutons sont friands ont été ajoutées à la ration.

La présence ou l'absence d'Oribates a été rigoureusement contrôlée tous les jours dans la nourriture ainsi distrıbuée.

A I total, 63 moutons ont été infestés de la fin de 1964 au début de 1966.

\section{Infestation.}

Elle ne souffre pas de difficultés. Les Oribates, une fois comptés et déterminés, sont administrés au mouton dans de l'eau « à la bouteılle ».

\section{RÉSULTATS}

Cinq tableaux donnent le résultat des infestations espèces, par espèces :

TABLEAUU N"Y

Ungrizetes reticulatus

\begin{tabular}{|c|c|c|c|c|}
\hline $\begin{array}{l}\text { Mouton } \\
\mathbb{N}^{\circ}\end{array}$ & $\begin{array}{l}\text { Origine } \\
\text { des } \\
\text { Oribates }\end{array}$ & $\begin{array}{c}\text { Nombre } \\
\text { d'Oribates } \\
\text { adm1nistrēs }\end{array}$ & $\begin{array}{l}\text { Nombre de } \\
\text { Striesia rencontrés } \\
\text { à l'autopsie }\end{array}$ & $\begin{array}{l}\text { Epoque des } \\
\text { Infestations }\end{array}$ \\
\hline 1 & $\mathrm{z}_{1}$ & 75 & 0 & Janvier 1965 \\
\hline 2 & $\mathrm{z}_{1}$ & 128 & 0 & \\
\hline 3 & $z_{1}$ & 112 & 0 & " \\
\hline Total & & 315 & 0 p. 100 & \\
\hline
\end{tabular}

TABLEAU N ${ }^{\circ}$ II

Africacarus calcaratus

\begin{tabular}{|c|c|c|c|c|}
\hline $\begin{array}{c}\text { Mouton } \\
\mathrm{N}^{\circ}\end{array}$ & $\begin{array}{c}\text { Origine } \\
\text { des } \\
\text { Oribates }\end{array}$ & $\begin{array}{c}\text { Nombre } \\
\text { d'Oribates } \\
\text { administrés }\end{array}$ & $\begin{array}{c}\text { Nombre de } \\
\text { Stiziesia rencontrés } \\
\text { à 1'autopsie }\end{array}$ & $\begin{array}{c}\text { Epoque des } \\
\text { infestations }\end{array}$ \\
\hline 1 & $\mathrm{z}_{1}$ & 17 & 0 & Janvier 1965 \\
2 & $\mathrm{z}_{1}$ & 224 & 14 & " Dëcembre 1964 \\
3 & $\mathrm{z}_{3}$ & 118 & 3 & Décembre 1964 \\
4 & $\mathrm{z}_{3}$ & 130 & $4,2 \mathrm{p} \cdot 100$ & \\
\hline Total & & 489 & & \\
\hline
\end{tabular}


Retour au menu

TABLEAU $N^{\circ}$ III

Galumna baloghi et Allogalumna pellucida

\begin{tabular}{|c|c|c|c|c|}
\hline $\begin{array}{l}\text { Mouton } \\
\mathrm{N}^{\circ}\end{array}$ & $\begin{array}{l}\text { Origine } \\
\text { des } \\
\text { Oribates }\end{array}$ & $\begin{array}{c}\text { Nombre } \\
\text { d'Oribates } \\
\text { administrês }\end{array}$ & $\begin{array}{l}\text { Nombre de } \\
\text { Sti legia rencontrés } \\
\text { à l'autopsie }\end{array}$ & $\begin{array}{l}\text { Epoque des } \\
\text { infestations }\end{array}$ \\
\hline 1 & $z_{1}$ & 10 & 0 & Janvier 1965 \\
\hline 2 & $z_{3}$ & 55 & 1 & Dëcembre 1964 \\
\hline 3 & $z_{3}^{3}$ & 31 & 0 & 1 \\
\hline 4 & $z_{3}^{3}$ & 42 & 36 & Mars \\
\hline 5 & $\mathrm{z}_{3}$ & 50 & 21 & Dêcembre 1964 \\
\hline 6 & $z_{3}$ & 52 & 0 & Janvier 1965 \\
\hline 7 & & 76 & o & Juillet $\quad 1965$ \\
\hline 8 & $z_{3}^{3}$ & 25 & 9 & Fëvrter 1965 \\
\hline 9 & $\mathrm{z}_{2}$ & 6 & 0 & Octobre 1965 \\
\hline Total & & 347 & $19,3 p \cdot 100$ & \\
\hline
\end{tabular}

TABLEAU N ${ }^{\circ} \mathrm{IV}$

Soheloribates perforatus

\begin{tabular}{|c|c|c|c|c|}
\hline $\begin{array}{l}\text { Mouton } \\
\mathrm{N}^{\circ}\end{array}$ & $\begin{array}{l}\text { Origine } \\
\text { des } \\
\text { Oribates }\end{array}$ & $\begin{array}{c}\text { Nombre } \\
\text { d'0ribates } \\
\text { administrés }\end{array}$ & $\begin{array}{l}\text { Nombre de } \\
\text { Stilesia rencontrés } \\
\text { à I'autopsie }\end{array}$ & $\begin{array}{l}\text { Epoque des } \\
\text { traitements }\end{array}$ \\
\hline 1 & $z_{2}$ & 50 & 9 & Octobre 1965 \\
\hline 2 & $z_{2}$ & 50 & 11 & $"$ \\
\hline 3 & $\mathrm{z}_{2}$ & 50 & 0 & Septembrel965 \\
\hline 4 & $z_{2}$ & 25 & 20 & Octobre 1965 \\
\hline 5 & $\mathrm{z}_{2}$ & 80 & 2 & 11 \\
\hline 6 & $z_{2}$ & 25 & 0 & " \\
\hline 7 & $z_{2}$ & 50 & 5 & " \\
\hline 8 & $\mathrm{z}_{2}$ & 35 & 0 & " \\
\hline 9 & $z_{3}$ & 313 & 3 & Mars \\
\hline 10 & $z_{3}$ & 167 & 0 & " \\
\hline 11 & $z_{3}$ & 329 & 0 & Fêvrier 1965 \\
\hline 12 & $z_{3}$ & 386 & 0 & " \\
\hline 13 & $\mathrm{z}_{3}$ & 379 & 0 & " \\
\hline 14 & $\mathrm{z}_{3}$ & 110 & 15 & Juin \\
\hline 15 & $z_{3}$ & 168 & 0 & $"$ \\
\hline 16 & $z_{3}$ & 429 & 5 & Décembre 1964 \\
\hline 17 & $\mathrm{z}_{3}$ & 250 & 27 & $"$ \\
\hline 18 & $\mathrm{z}_{3}$ & 140 & 0 & Mars \\
\hline 19 & $z_{3}$ & 110 & 8 & 11 \\
\hline 20 & $\mathrm{z}_{3}$ & 185 & 1 & Décembre 1964 \\
\hline 21 & $z_{3}$ & 130 & 0 & " \\
\hline 22 & $\mathrm{z}_{2}$ & 8 & 0 & 1966 \\
\hline 23 & $\mathrm{z}_{2}$ & 22 & 4 & " \\
\hline Total & & 3491 & 2,9 p.100 & \\
\hline
\end{tabular}


TABLEAU $\mathbb{N}^{\bullet} \nabla$

Scheloribates fimbriatus var. Africarus

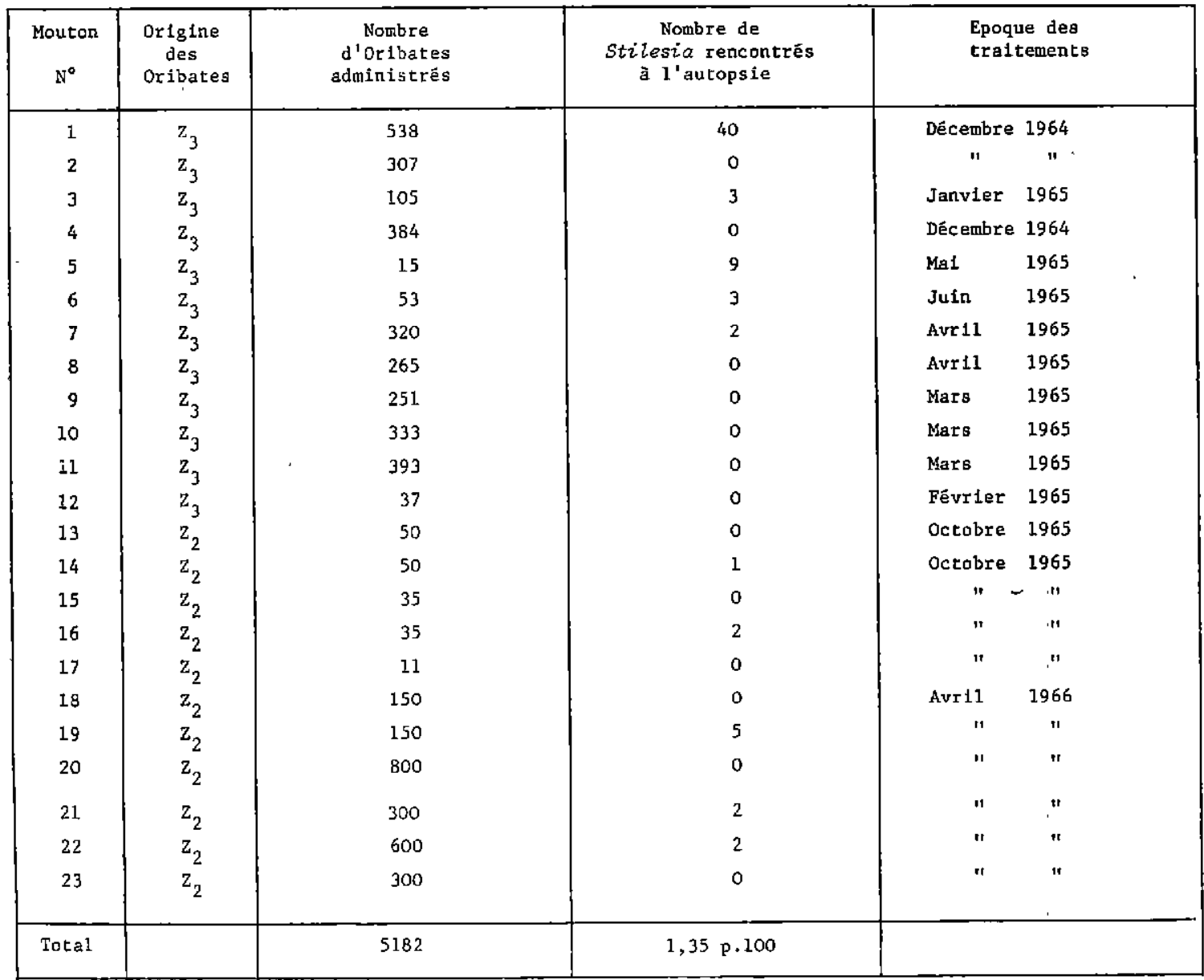

60 Les Oppia sont incapables de donner au mouton Stilesio globipunctota et un essai, tenté à partir de 210 de ces Acariens, est resté complètement négatif.

\section{DISCUSSION}

10 En définitive, les œufs de Stilesio globipunctata sont susceptibles d'être absorbées par un grand nombre d'Oribates où ils évolueront en donnant des Cysticercoides. Ce sont, compte tenu de ce qui a été écrit précédemment (GRABER ef GRUVEL, 1964) :

Scheloribates perforatus.

Scheloribates parvus conglobatus.

Scheloribates fimbriatus ofricanus.
Galumna baloghi.

Allogolumno pellucida.

Africacorus calcoratus.

Au total donc, pour l'instant, 4 genres et six espèces.

II n'y a aucune spécificité de vecteurs dans le cas de ce Cestode et, selon les populations, plusieurs espèces d'Oribateš peuvent remplir ce rôle. Il en est de même avec Moniezio expansa, puisque des Cysticercoïdes de Moniezia ont été observées chez trois espèces différentes en Amérique (KATES et RUNKEL, 1948), sept espèces en Hongrie (KASSAl et MAHUNKA, 1964) et sept espèces également en Bulgarie (BANKOV, 1965). 
20 De cette abondance de vecteurs, il résulte que les possibilités de transmission au mouton de Stilesia globipunctota sont multiples et existent pratiquement toute l'année, ce que laissait déjà supposer les premières observations faites (GRABER ef GRUVEL, 1964).

L'infestation d'octobre à juillet pourraił être schématisée ainsi dans la région de Fort-Lamy :

TABLEAU N"Vi

Infestation du mouton à partir d'Oribates porteurs de Cysticercoldes de Stizesia.

\begin{tabular}{|c|c|}
\hline Mo1s & Vecteurs \\
\hline Octobre & $\begin{array}{l}\text { Scheloribates perforatus } \\
\text { Scheloribates fimbriatus af ricarus }\end{array}$ \\
\hline Novembre & $\begin{array}{l}\text { Af ricacarus catearatus } \\
\text { Scheloribates perforatus }\end{array}$ \\
\hline Décembre & $\begin{array}{l}\text { Africacarus calcaratus } \\
\text { Scheloribates perforatus } \\
\text { Galumn baloghi } \\
\text { Allogalumra pellucida } \\
\text { Scheloribates fimbriatus africans }\end{array}$ \\
\hline Janvier & $\begin{array}{l}\text { Soheloribates fimbmiatus africanus } \\
\text { Schezoribates perforatus }\end{array}$ \\
\hline Février & $\begin{array}{l}\text { Gatumna balogin } \\
\text { Schelaribates perforatus } \\
\text { Altogalumna pellucida }\end{array}$ \\
\hline Mars & $\begin{array}{l}\text { Schetoribates perforatus } \\
\text { Galumra balogin } \\
\text { Allogalumn pellucida }\end{array}$ \\
\hline Avr11 & $\begin{array}{l}\text { Schelombates perforatus } \\
\text { Schetoribates fimbriatus africanus }\end{array}$ \\
\hline Maî & $\begin{array}{l}\text { Seheloribates perforatus } \\
\text { Scheloribates fimbriatus africarus }\end{array}$ \\
\hline Jufn & $\begin{array}{l}\text { Schetoribates perforatus } \\
\text { Soheloribates parvus conglobatus }{ }^{+} \\
\text {Scheloribates fimbriatus afriaans }\end{array}$ \\
\hline
\end{tabular}

+ Volr expërience précêdente (Graber, Gruvel,1964)

L'autopsie de 250 moutons effectuée durant la même période montre que, si la masse parasitare (poids moyen mensuel de Stilesia en grammes) varie peu, le nombre d'anımaux atteints subit des fluctuations un peu plus amples, les pourcentages les plus bas correspondant aux époques où les Acariens, pour diverses raisons (terrains détrempés par les pluies en fin d'hivernage; grosses chaleurs du printemps), sont moins nombreux à la surface du sol (Graphique no 1).
Chaque mois, la présence dans les pâtures de deux ou de plusieurs espèces d'Oribates potentiellement parasifées assure donc la pérennité de l'infestation par Stilesia globipunctato.

$3^{\circ} \mathrm{La}$ comparaison entre les pourcentages d'Acariens hébergeant des Cysticercoïdes au cours des années 1964-1965 et 1964-1966 est particulièrement instructive : (tableau Vil).

Dans les conditions naturelles, les meilleurs vecteurs paraissent appartenir à la famille des Galumnidae et aux genres Galumno et Allogalumna. Leur petite tailie (de 310 d̀ $370 \mu$ en moyenne) ne semble pas constituer un obstacle à l'absorption des œufs de Stllesia.

Viennent ensuite des Acariens de la famille des Cerotozefidae.

Quant aux Scheloribates, même dans des conditıons expérimentales, ils se comportent comme des vecteurs apparemment assez médiocres, parfois purement accidentals (S. parvus conglobatus). L'un d'entre eux cependant, Scheloribates perforatus compense ce désavantage par son abondance, sa très large dispersion et par le fait qu'il forme souvent des colonies importantes : c'est donc lui, de préférence aux espèces précédentes, que le mouton aura le plus de chance de rencontrer sur les herbes des pâturages.

\section{CONCLUSIONS}

10 De 1964 à 1966, les auteurs ont poursuivi l'étude des Oribates vecteurs de Stilesia globi punctata (RIVOLTA, 1874) des ovins du Tchad.

$2^{\circ}$ A partir d'Oribates naturellement infestés, prélevés à l'intérieur et à l'extérieur de la concession du Laboratoire de Farcha, il a été possible de démontrer que six espèces différentes pouvaient transmettre cet Anoplocephalidae à des moutons préalablement déparasités (63). Ce sont : Scheloribates perforatus (WALLWORK, 1964), Scheloribates parvus Van Pletzen conglobatus (WALLWORK, 1964), Scheloribates fimbriatus Thor africonus (WALLWORK, 1964), Golumna bologhi (WALLWORK, 1965), Allogolumno pellucida (WALLWORK, 1965), Africacorus colcoratus (WALLWORK, 1965),

3o Des observations faites d'octobre à juillet indiquent qu'il peut exister chaque mois dans les pâtures au mınimum deux et au maximum cinq espèces hébergeant des Cysticercoides de 
Graphique I STILESIA GLOBIPUNCTATA

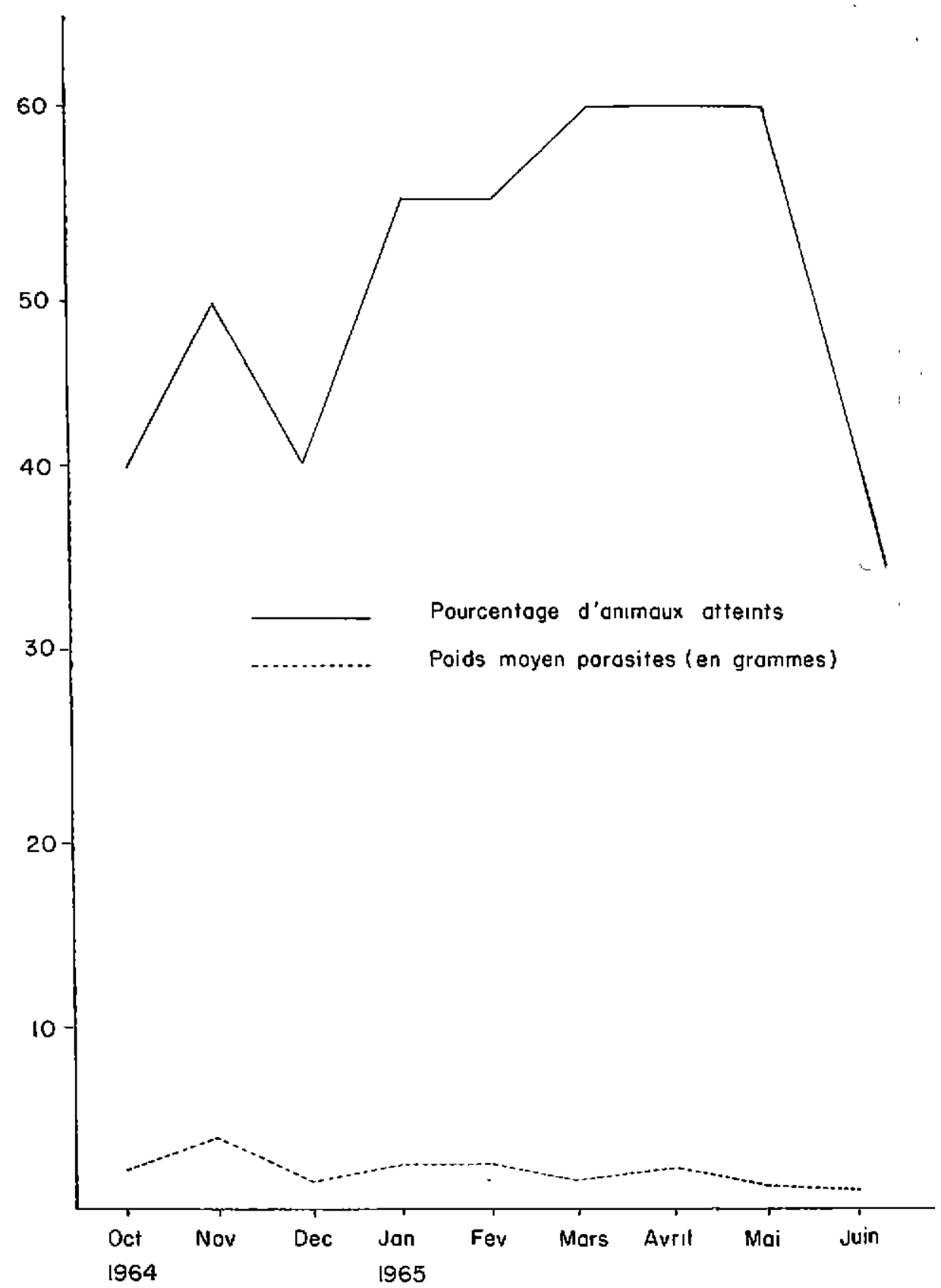


TABLEAU N $N^{\circ} \mathrm{VII}$

Pourcentage d'Acarlens hêbergeant des Cysticercoïdes

\begin{tabular}{|c|c|c|}
\hline Oribates vecteurs & Octobre 1963 à Juin 1964 & Octobre 1964 à MaI 1966 \\
\hline $\begin{array}{l}\text { Unouizetes reticulatus } \\
\text { Oppia spp. } \\
\text { Afmicacarus calcaratus } \\
\text { Galumna baloghi } \\
\text { Allogalumn pellucida } \\
\text { Scheloribates perforatus } \\
\text { Scheloribates fimbriatus } \\
\text { afmeans } \\
\text { Scheloribates parvus } \\
\text { conglobatus }\end{array}$ & $\begin{array}{c}- \\
- \\
0,87 \mathrm{p} \cdot 100 \\
- \\
3,95 \mathrm{p} \cdot 100^{+} \\
- \\
0,2 \mathrm{p} \cdot 100\end{array}$ & $\begin{array}{c}0 \text { p.100 } \\
0 \quad " \\
4,2 \quad " \\
19,3 \quad " \\
2,93 " \\
1,35 "\end{array}$ \\
\hline
\end{tabular}

$+\Rightarrow$ un certain nombre de Scheloribates avalent étê infestés artificiellement.

Stilesia globipunctata, ce qui assure la pérennité ef la constance de l'infestation chez le mouton. Les autopsies effectuées pendant la même période sur 250 moutons originarres de la région de Fort-Lamy confirment ce point de vue.

40 Les meilleurs vecteurs appartiennent d̀ la famille des Galumnidae ( $G$. baloghi et $A$. pellucida). Viennent ensuite des Ceratozetidae (A. calcaratus). Scheloribates perforatus, l'espèce la plus commune, apparaît comme un hôte intermédiaire assez médiocre qui compense son faible taux d'infestation par une très large dispersion sur le terraın, ce qui la met, tout compte fait, plus souvent que les autres espèces en contact, dans les pâturages, avec l'hôte définitif de Stilesia globipunctato.

\section{Instihut d'Elevage et de Médecıne Vétérinaire des pays Tropicoux \\ Laboratore de Forcha - Fort-Lamy - République du Tchod}

\section{SUMMARY}

The vectors of Stilesio globipunctata (Rivolta, 1874) of sheep

The authors have transmired Stilesia globipunctata (RIVOLTA, 1874) to parasite free sheeps from the folloving Oribatidae Scheloribates perforatus (WALLWORK, 1964), Scheloribates parvus Van Pletzen conglobatus (WALLWORK, 1964), Scheloribates fimbriotus Thor africanus (WALLWORK, 1964) Galumno pellucida (WALLWORK, 1965) et Africacarus caicaratus (WALLWORK, 1965(.

In each month, there is in the grasslands at least two and at most five different species, which are able to harbour the Cysticercordes of this Anoplocephalidae, and therefore to keep the perennity and the persistence of the infestation in sheep.

The best vectors seen belong to the Golumnidae family Scheioribotes perforatus compensates a comparatively low rate of infestation by a widespread prevalence and a large number of parasites. Therefore, the sheep final host, is able to ingest it often than Ihe others species which can act as vectors. 


\section{RESUMEN}

Los vectores de Stilesia globipunciafo (Rivolta, 1874) en la oveja

A partir de Los Oribatos siguientes: Scheioribates perforotus (WALLWORK, 1964), Scheloribates parvus Van Pietzen conglobatus (WALLWORK, 1964), scheloribotes fimbriotus Thor ofricanus (WALLWORK, 1964), Galumna balogh (WALLWORK, 1964), Allogalumna pellucida (WALLWORK, 1965) y africacarus calcaratus (WALLWORK, 1965), los autores acertaron transmitir stilesio giobipunctato (RIVOLTA, 1874) en ovejas deparasitadas (63).

En los pastos se encuentran cada mes al minimo dos y al maximo cinco especies susceptibles de alojar las cisticercoidos de este Anaplocephalidae, to que asegura la permanencia de la infestación en la oveja.

Pertenecen los mejores vectores a la familia de Ios Galumnidae. Scheloribates perforatus tiene un termino medio de infestación relativamente poco imporłante pero está muy dispersado en gran cantidad. Asi, la oveja, huésped definitıvo, puede absorberle más frecuentemente que las otras especies pudiendo ser vectores.

\section{BIBLIOGRAPHIE}

BANKOV (D.). - The biology of Moniezio and the epidemiology of Monieziosis in Bulgaria. Vet. Med. Naukı. Sofia, 1965, 283-294.

GRABER (M.) et GRUVEL (J.). - Transmission au mouton de Stilesia globipunctata Rivolia 1874 (Cestoda, Anoplocephalidae), à partir de divers Acariens Oribates. C. R. Acad. sci. Paris, 1964, 259, 4811-4814.

GRABER (M.) et GRUVEL (J.). - Note préliminaire concernant la transmission de Stilesia globipuncfata (Rivolta, 1874) du mouton par divers Acariens Oribates. Rev. Elev. Méd. Vét. Pays Trop., 1964, 17, 3, 467476.

GRABER (M.) et SERVICE (J.). - Le téniasis des bovins et des ovins de la République du Tchad. Quelques données épidémiologiques intéressant les zones sahéliennes. Rev. Elev. Méd. Vét. Pays Trop., 1964, 17, 3, 491-521.

GRABER - (M.). - Helminthes et Helminthiases faisant obstacle à l'amélioration de la production ovine en République du Tchad. Monographie, 1965, 158 pp.
KASSAI (T.) et MAHUNKA (S.). - Vectors of Moniezia. Mogy. Allatorv. Lop., 1964, 19. 531-538.

KASSAI (T.) et MAHUNKA (S.), - Studies on tapeworms in ruminants. Il. Oribatides as intermediate hosts of Moniezia species. Acto Vet. Hung. Budopest, 1965, 15, 221-249.

KATES (K. C.) and RUNKEL (C. E.). - Observations on Oribatide mite vectors on pasiure with a report of several new vectors. U.S. Proc. Helm. Soc. Wash., 1948, 15, 1, 19-33.

TANDON (R. S.). - Observations on the lifehistory of the Anoplocephalidae Cestode, Stilesia globipunctato Rivolta, 1874 (Subfamily Thysonosominae), a common parasite of ruminanis in India. Parassitologia, 1963, 5, 3, 183-187.

WALLWORK (J. A.), 一 Some Oribatei (Acari : cryptosfigmafa) from Tchad. Rev. Zool. Bot. ofr., 1964, LXX, 3/4, 353-385.

WALLWORK (J. A.). - Some Oribatei (Acari : cryptostigmata) from Tchad. Rev. Zool. Bot. Afr., 1965, LXII, 1/2, 83-108. 